\section{MANIPULATION OF EPITHELIAL-TO-MESENCHYMAL TRANSITION REVEALS METASTATIC BREAST CANCERS SUPPORT IMMUNE SUPPRESSION VIA HEME METABOLISM}

Michelle Williams*, Sabrina Hafeez, Jessica Christenson, Nicole Spoelstra, Jill Slansky, Jennifer Richer. University of Colorado Anschutz Medical Campus, Aurora, CO, USA

Background Oncogenic epithelial-to-mesenchymal transition (EMT) enhances triple negative breast cancer (TNBC) aggressiveness and immune suppression. To identify alternative immunotherapy targets for metastatic TNBC, we reversed EMT in mesenchymal-like human TNBC and mouse mammary carcinoma models by restoring the micro-RNA-200c. This approach identified several tumor cell metabolizing enzymes with potential immune modulatory functions such as heme oxygenase-1 (HO-1). HO-1 converts heme to bilirubin, an established immune suppressor in other diseases that has never been tested as such in cancer. We hypothesize that tumor cellHO-1 activity and subsequent bilirubin secretion enhance TNBC metastasis by supporting a pro-tumor immune microenvironment (figure 1 ).

Methods We tested the impact of tumor cell-HO-1 and bilirubin on macrophage immune suppression and efferocytic capacity (engulfment of dead tumor cells) using qRT-PCR, flow cytometry and live cell imaging. Human and mouse macrophages were analyzed after treatment with exogenous bilirubin or bilirubin-depleted conditioned medium collected from tumor cells treated with tin mesoporphyrin (SnMP), an enzymatic HO-1 inhibitor. Primary tumor growth and lung metastatic burden were observed in syngeneic mice harboring $\mathrm{HO}-$ 1 depleted 66Cl-4 mammary tumors (shRNA). Breast cancer specimens were analyzed via CIBERSORT to predict immune cell abundance in patients with high versus low levels of heme metabolism genes.

Results Macrophages cultured with conditioned medium from tumor cells treated with the HO-1 inhibitor SnMP demonstrated a $35-65 \%$ decrease in immune suppressive genes (Arg1, Cd274, Tgfb1) compared to those treated with control conditioned medium. This effect was rescued by exogenous treatment with $2.5 \mu \mathrm{M}$ bilirubin. Direct bilirubin treatment enhanced macrophage PD-L1 mRNA and protein expression by at least 6-fold. In contrast, bilirubin decreased expression of macrophage efferocytosis genes (Mertk, Tyro3) by at least $50 \%$, resulting in decreased efferocytic capacity. To test whether bilirubin supports tumor progression via modulation of macrophages, we evaluated tumor growth and metastasis after tumor cell-HO-1 depletion. While mice with shHO1 tumors had enhanced primary tumor growth compared to those with shCnt tumors, HO-1 depletion decreased lung metastatic capacity. Although immune cell infiltration and activation is currently underway in this mouse model, CIBERSORT analysis revealed that breast cancer specimens with high levels of heme metabolism genes have a predicted increase in M2 macrophage presence.

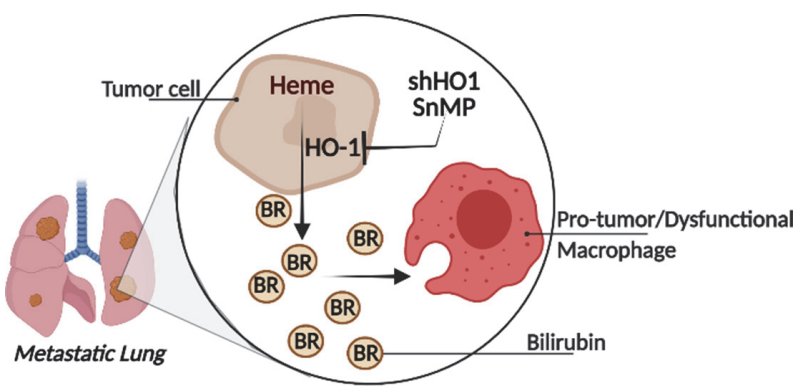

Abstract 890 Figure 1 Project model

Heme oxygease-1 (HO-1) breaks down heme into immune modulatory products such as bilirubin (BR). We demonstrated that tumor cell-secreted BR may enhance TNBC lung metastasis by supporting macrophage immune suppression and dysfunction. This can be blocked by genetic (shRNA) or pharmacologic (SnMP) inhibition of HO-1 in TNBC cells. Figure made with biorender.com.

Conclusions Tumor cell-HO-1 may support immune cell suppression and dysfunction during breast cancer metastasis via bilirubin. Since HO-1 inhibitors including SnMP are FDA approved for treatment of other diseases, these findings could rapidly be translated to provide an additional immunotherapy for metastatic TNBC.

http://dx.doi.org/10.1136/jitc-2021-SITC2021.890 